\title{
An Optimal Design of the Compact CRLH-TL UWB Filter Using a Modified Evolution Strategy Algorithm
}

\author{
Seung-Hun Oh*, Chao Wu*, Tae Kyung Chung * and Hyeong-Seok Kim ${ }^{\dagger}$
}

\begin{abstract}
This paper deals with an efficient optimization design method of a compact ultra wideband (UWB) filter which can improve the characteristics of the filter. The Evolution Strategy (ES) algorithm is adopted for the optimization and modified to suppress the ripple by inserting an additional step to the ES scheme. The algorithm has the ability to control the ripple of an insertion loss in a passband as a modified approach. During the modified ES, a structure of initial shape is changed a lot, while includes the stepped impedance (SI) and the composite right/left handed transmission line (CRLH-TL). And an optimized filter satisfies the UWB specifications on the stopband and passband with an acceptable insertion loss. The filter achieves a much developed shape, the size of $15 \times 14 \mathrm{~mm}$, the $3 \mathrm{~dB}$ bandwidth from 2.7 to $10.8 \mathrm{GHz}$, the flat insertion-loss less than $1 \mathrm{~dB}$, the wide stopband with $12 \sim 20 \mathrm{GHz}$, and an acceptable return loss.
\end{abstract}

Keywords: CRLH-TL, UWB Filter, SI, ES, Ripple, Step of suppressing the ripples

\section{Introduction}

Ever since the release of the UWB communication to the public, many researches have been carried out on components. As one of many researches, the band-pass filter has been developed. Most of the previous UWB filters have used the half- or quarter-wavelength method, which limits the size-reduction $[1,2]$.

CRLH-TL metamaterial UWB filters were proposed to resolve this limit in miniaturization [3-8]. These filters result from a feature that a size of the CRLH-TL resonator is just a fraction of the wavelength.

Using the CRLH-TL resonator is helpful to making the entire geometry compact. However, elements in a small structure are too much complicatedly coupled, which turns out hard to control either a passband or stopband $[3,4]$. There is no closed form for the design, and it makes the process complicated.

The number of the elements increases such as higher order coupling and addition of a lowpass filtering block. If the filter is designed through the unsystematic process, a design time becomes longer and the unnecessary process occurs [5-8].

A conspicuous problem occurring from the unsystematic filter-design process is a ripple in the passband due to the impedance mismatch. The ripple is an unstable insertion loss in the passband, and will lead to the degradation in the overall system performance [9].

In order to design a filtering device automatically and

$\dagger$ Corresponding Author: School of Electrical and Electronics Eng., Chung-Ang University, Korea. (kimcaf2@cau.ac.kr)

* School of Electrical and Electronics Engineering, Chung-Ang University, Korea. (trydent0@naver.com, wuchao19910527@ hotmail. com, tkchung@cau.ac.kr)

Received: September 19, 2014; Accepted: November 8, 2014 optimally, a great number of optimization algorithms have been applied. Most of them take many sample points in the bandwidth to define a cost function [10]. In particular, when a very wide-band filter is in need, an extended number of sample points are taken in the band and their function values are averaged in handling the cost function, and it misses a few ripple points of the impedance mismatch. Therefore, the steps to check and treat the ripple points should be included in the wide-band filter design.

This paper suggests an efficient optimization design method of a compact UWB filter by using a modified ES algorithm. The modified ES is achieved by inserting a new step of handling the slopes of the target graph to the conventional scheme to suppress the ripples in the band.

\section{SI-COMBINED CRLH-TL FILTER}

\subsection{Design of the CRLH-TL UWB filter}

The CRLH-TL is a composite form between the right handed transmission line (RH-TL) as the conventional transmission line and the left handed transmission line ( $\mathrm{LH}-$ TL) with the negative propagation constant. Combining the $\mathrm{LH}$ and $\mathrm{RH}$ regions enables the CRLH structure to have a wideband. It can be modeled as follows [4-8].

Fig. 1(a) is the equivalent circuit of the conventional periodic CRLH-TL. Series inductance $L_{R}$ and shunt capacitance $C_{R}$ account for the RH-TL. Meanwhile, series capacitance $C_{L}$ and shunt inductance $L_{L}$ are essential to the LH-TL. The formulae of the important frequency points are summarized as eqn. (1), (2), and (3).

In eqn. (1) (3), $f_{0}, f_{L}$ and $f_{R}$ mean the center frequency, and the negative first resonance and positive first resonance 


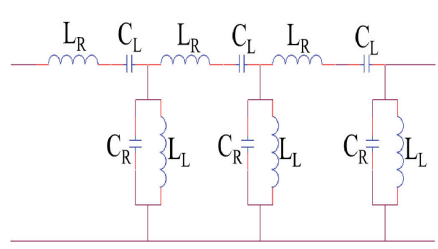

(a)

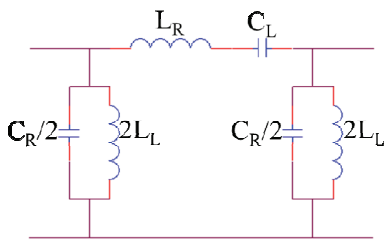

(b)
Fig. 1. Circuit models of the CRLH-TL (a) periodic circuit, (b) $\pi$-type unit-cell circuit

frequencies. The $\mathrm{L}_{\mathrm{L}}, \mathrm{L}_{\mathrm{C}}, \mathrm{C}_{\mathrm{L}}$ and $\mathrm{C}_{\mathrm{C}}$ are the circuital elements of CRLH-TL. The periodic structure like Fig. 1(a) ends up being long in size, and the $\pi$-type unit-cell in Fig. $1(b)$ is adopted as the basis for a size-reduced filter.

$$
\begin{gathered}
f_{0}=f_{\text {Series }}=f_{\text {Shunt }}=\sqrt{f_{L} f_{R}} \\
f_{L}=\frac{1}{2 \pi \sqrt{L_{L} C_{L}}}, f_{R}=\frac{1}{2 \pi \sqrt{L_{R} C_{R}}} \\
f_{\text {Series }}=\frac{1}{2 \pi \sqrt{L_{R} C_{L}}}, f_{\text {Shunt }}=\frac{1}{2 \pi \sqrt{L_{L} C_{R}}}
\end{gathered}
$$

When Fig. 1(b) is physically realized, its preliminary form can be made as Fig. 2(a). The short-circuited stub is a good candidate for the shunt inductance and the interdigital line is chosen for the series capacitance and inductance [11]. There is the interdigital line composed of 6 fingers and enclosed by a loop shorted at the bottom, which has the $\mathrm{RH}$ resonance far away from the $\mathrm{LH}$ region and the center frequency of $6 \mathrm{GHz}$. The frequency response of Fig. 2(a) is obtained by the full-wave simulator as shown in Fig. 2(b).

\subsection{SI as a lowpass filtering}

Seeing its $\mathrm{S}_{21}$ as in Fig. 2(b), the frequency response does not have the stopband at the frequency beyond 10.8 GHz. If the upper segment of the shorted loop is modified to an SI path, it can work as a lowpass filter.

Before accomplishing the ultimate UWB bandpass filter, a work is done first to obtain the stopband by handling the (a)

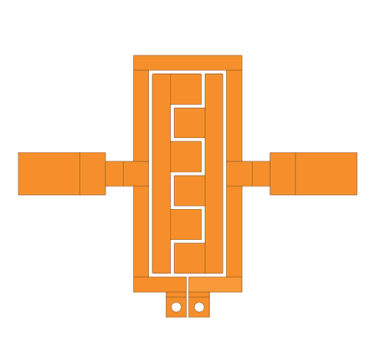

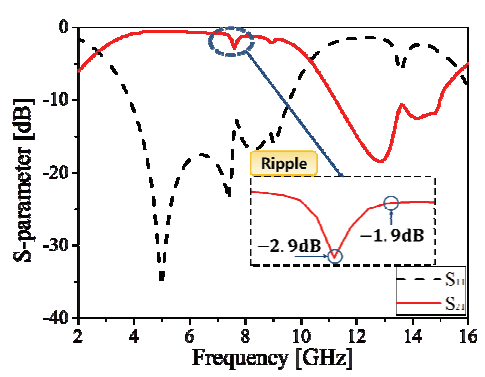

(b)
Fig. 2. Initially proposed shape and S-parameters; (a) Initially proposed shape; (b) S-parameters of the proposed shape

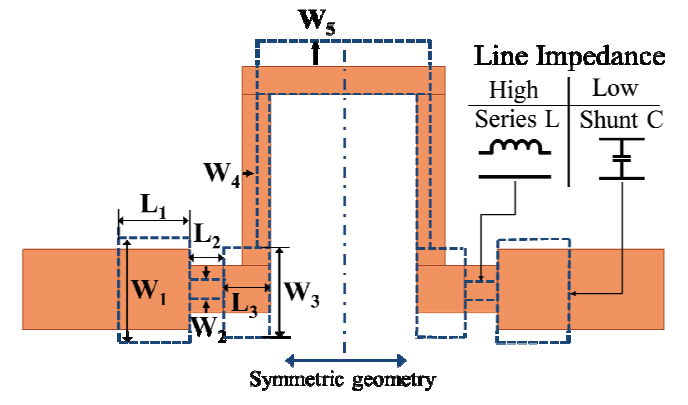

(a)

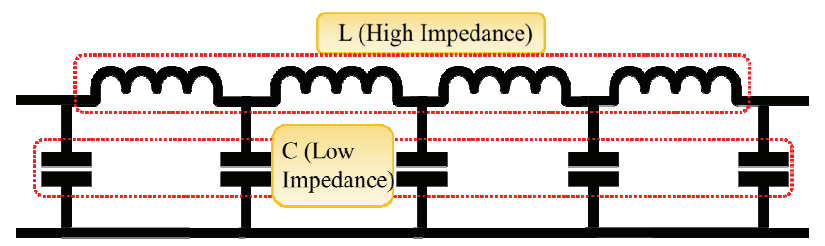

(b)

Fig. 3. SI path of the CRLH-TL filter and its electrical meaning (a) Top-view of the SI path segmentation with lowpass filtering (b) Its equivalent circuit

SI path [9, 12]. To use the SI path as a lowpass filtering block, it should be divided into segments as in the top-view of the SI path of Fig. 3(a). The segments correspond to inductance (L) and capacitance (C) elements in a lowpass filter as Fig. 3(b). Shunt C's and series L's are wide and narrow segments, respectively. Once the specifications of the lowpass filter are given, shunt C's and series L's can be calculated and they are converted to the physical dimensions by way of the transmission-line theory, or vice versa.

\subsection{The shape of the SI combined CRLH-TL and geometrical dimensions to be optimized}

The SI path is incorporated to the shorted loop enclosing the interdigital lines as follows to become a complete filter.

Fig. 4 is an initial shape of the filter and the geometrical parameters will be optimized to have the satisfactory results of the passband and stopband. The widths and

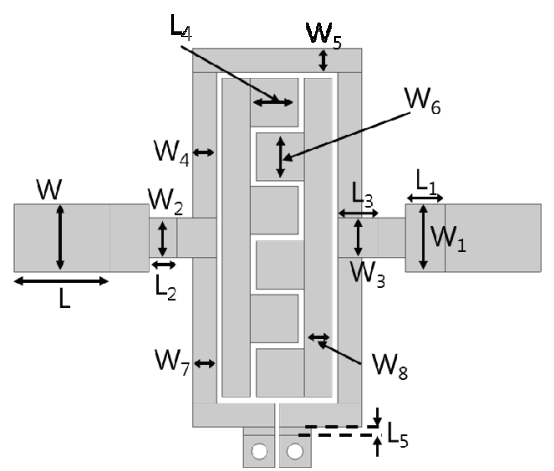

Fig. 4. The shape and physical dimensions for the optimized design 
lengths of the nine segments in Fig 4 are obtained as (W, $1.6 \mathrm{~mm}),(\mathrm{W} 1,1.6 \mathrm{~mm}),(\mathrm{W} 2,1 \mathrm{~mm})(\mathrm{W} 3,1 \mathrm{~mm}),(\mathrm{W} 4$, $0.6 \mathrm{~mm}),(\mathrm{W} 5,0.6 \mathrm{~mm}),(\mathrm{W} 6,1.2 \mathrm{~mm}),(\mathrm{W} 7,0.6 \mathrm{~mm}),(\mathrm{W} 8$, $0.7 \mathrm{~mm}),(\mathrm{L}, 2.8 \mathrm{~mm}),(\mathrm{L}, 0.6 \mathrm{~mm}),(\mathrm{L} 2,0.6 \mathrm{~mm}),(\mathrm{L} 3$, $0.5 \mathrm{~mm})$ and $(\mathrm{L} 4, .1 .2 \mathrm{~mm})$.

\section{Es Optimization Of Si-Combined Crlh Uwb Filter With Mitigating The Ripples}

\subsection{Proposed step of suppressing the ripples in the pass-band}

Since the CRLH UWB filter structure has complicated electromagnetic interaction from one part to another, there is no analytic design formula. Moreover, the SI path and loop is added to the interdigital lines resulting in extra coupling, and the design becomes more complex and it is highly probable to have the passband ripples due to impedance mismatch.

The ripple of $\mathrm{S}_{21}$ in Fig. 2(b) clearly shows the signal becomes weaker and the return loss increase at $7.8 \mathrm{GHz}$. So, the ripple larger than $0.5 \mathrm{~dB}$ should be spotted in the passband and mitigated. The shaded box in Fig. 5 presents the procedures to search the location and level of the ripple by detecting the change in the $\mathrm{S}_{21}$.

The process is explained in detail as follows. The passband is segmented into sample points. The slope of the $\mathrm{S}_{21}$ curve is evaluated as the sample point progresses, and it stores $\left|\mathrm{S}_{21}\left(f_{a}\right)\right|_{\mathrm{dB}}$ at $f_{a}$ when the slope changes from negative to positive. Immediately after $f_{a}$, when the slope varies from positive to negative, the location is stored as $f_{b}$ with $\left|\mathrm{S}_{21}\left(f_{b}\right)\right|_{\mathrm{dB}}$. Simultaneously, $\left|\mathrm{S}_{21}\left(f_{b}\right)\right|_{\mathrm{dB}}$ and $\left|\mathrm{S}_{21}\left(f_{a}\right)\right|_{\mathrm{dB}}$ are compared and if their difference exceeds a certain level $\mathrm{R} 0$ (e.g. $0.2 \mathrm{~dB}$ ), it is sensed as the ripple which will be suppressed by the optimization of the filter.

\subsection{Filter optimization using the ES with suppressing ripples}

As the shape and inter-element coupling of the proposed filter have a high degree of complexity, it is hard to decide the optimal geometry and physical dimensions analytically. This motivates us to use a full-wave solver and combine it with an automatic optimization method. Though there are several stochastic optimization methods, in order to reduce the work time, the ES is chosen, which is simple to use and combine with the full-wave solver [13]. Particularly, $(1+1)$ ES is adopted. The field and s-parameters are calculated by the HFSS, a commercial EM solver, and we write an EXCEL program linking the macro interactive function of the EM solver to the ES optimization framework [14].

The optimization of the present UWB filter should make low insertion loss $\left(\mathrm{S}_{21}\right)$ and return loss $\left(\mathrm{S}_{11}\right)$ in the passband and a high attenuation $\left(\mathrm{S}_{21}\right)$ in the stopband. Therefore, this requirement is expressed as the objective function that consists of the passband insertion- and return losses and the suppression level in the stopband.

$$
\begin{gathered}
F_{t}=\alpha_{1} F_{S_{11}}^{\text {Band } 1}+\alpha_{2} F_{S_{21}}^{\text {Band } 1}+\alpha_{3} F_{S_{21}}^{\text {Band } 2} \\
\alpha_{m} \in[0,1], \quad m=1,2,3
\end{gathered}
$$

where $\alpha$ is the weighting factor. The weighting factor for the term with the largest value among the square terms in (4) becomes 1, and weighting factors for other terms are set such that each term can be almost equal to the largest value.

The terms in eqn. (4) are given as

$$
\begin{aligned}
& F_{S_{11}}^{\text {Band } 1}=\frac{1}{N_{S_{11}}} \sum_{f=f_{1}}^{f_{2}} \max \left(0, S_{11}(f)-S_{O 1}\right) \\
& F_{S_{21}}^{\text {Band } 1}=\frac{1}{N_{S_{21}}} \sum_{f=f_{1}}^{f_{2}} \max \left(0, S_{O 2}-S_{21}(f)\right) \\
& F_{S_{21}}^{\text {Band } 2}=\frac{1}{N_{S_{21}}} \sum_{f=f_{3}}^{f_{4}} \max \left(0, S_{21}(f)-S_{O 3}\right)
\end{aligned}
$$

In eqn. (5) (7), $\mathrm{N}$ is the number of the sample frequency $f, \mathrm{~S}_{11}(f)$ and $\mathrm{S}_{21}(f)$ in Bandl mean the insertion- and return losses at the sample frequency $f$ in the passband. And, $\mathrm{S}_{21}(f)$ in Band2 is the level of suppression in the stopband. These s-parameter values are compared to the targets as $\mathrm{S}_{\mathrm{O} 1}, \mathrm{~S}_{\mathrm{O} 2}$, and $\mathrm{S}_{\mathrm{O} 3}$. Band 1 and Band 2 range from $f_{1}$ to $f_{2}$, and from $f_{3}$ to $f_{4}$, respectively. Also, there are $\mathrm{Ns}_{11}$ and $\mathrm{Ns}_{21}$ sample points in Band 1 and Band 2 in that order. In the cost functions, the target $\mathrm{S}$-parameter values are set as $\mathrm{S}_{\mathrm{O} 1}=-1.3 \mathrm{~dB}, \mathrm{~S}_{\mathrm{O} 2}=-10 \mathrm{~dB}$, and $\mathrm{S}_{\mathrm{O} 3}=-20 \mathrm{~dB}$ with $f_{1}=3.1$ $\mathrm{GHz}, f_{2}=10.6 \mathrm{GHz}, f_{3}=12 \mathrm{GHz}$, and $f_{4}=20 \mathrm{GHz}$.

As is commonly done in other problems, the cost function in Eqn. (5) (7) uses the average of all the errors

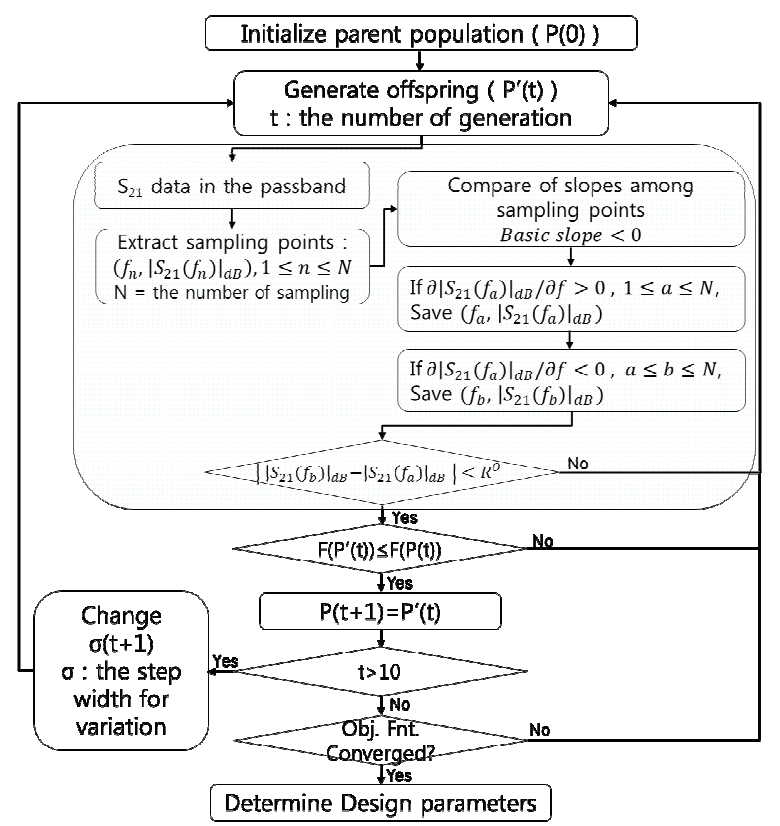

Fig. 5. Flowchart of the modified ES 


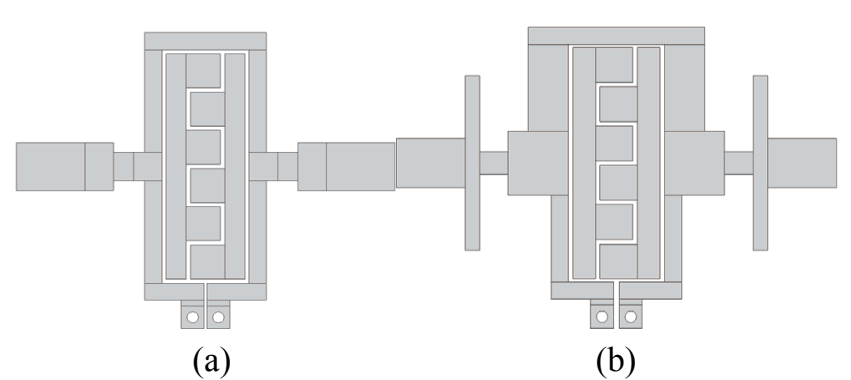

Fig. 6. The initial shape changes by the proposed method (a) Initial (b) ES with the ripple suppression

Table 1. Optimized Values of Parameters [unit : mm]

\begin{tabular}{c|c|c|c|c|c|c|c}
\hline State & $\mathrm{W}$ & $\mathrm{W}_{1}$ & $\mathrm{~W}_{2}$ & $\mathrm{~W}_{3}$ & $\mathrm{~W}_{4}$ & $\mathrm{~W}_{5}$ & $\mathrm{~W}_{6}$ \\
\hline Initial & 1.6 & 1.6 & 1 & 1 & 0.6 & 0.6 & 1.2 \\
Only ES & 1.4 & 5.86 & 0.69 & 1.58 & 1.28 & 0.72 & 1.21 \\
ES with SR & 1.7 & 5.8 & 0.8 & 2.2 & 1.42 & 0.6 & 1.2 \\
\hline State & $\mathrm{W}_{7}$ & $\mathrm{~W}_{8}$ & $\mathrm{~L}$ & $\mathrm{~L}_{1}$ & $\mathrm{~L}_{2}$ & $\mathrm{~L}_{3}$ & $\mathrm{~L}_{4}$ \\
\hline Initial & 0.6 & 0.7 & 2.8 & 0.6 & 0.6 & 0.5 & 1.2 \\
Only ES & 0.48 & 0.67 & 2.47 & 0.38 & 1.17 & 1.45 & 1.44 \\
ES with SR & 0.6 & 0.89 & 2.39 & 0.5 & 1 & 1.5 & 1.28 \\
\hline
\end{tabular}

at the sample points. Because of this averaging, the optimization process possibly misses the ripples that occur off the sample points. If the ripples are not taken into consideration, the main purpose cannot be fulfilled. Hence, the step suggested in the previous section is inserted in the ES optimization to suppress the ripples after spotting their positions and levels, which is defined a modified ES algorithm in this paper.

In Fig. 5, the modified ES algorithm governs the entire optimization where the s-parameters are calculated by the EM solver and evaluated in terms of the cost function in each iteration. Different from other approaches, the slope of $\mathrm{S}_{21}$ and ripple level are checked and controlled to meet the objectives.

After the use of this algorithm, the initial shape of the SI-combined CRLH UWB filter has changed from Fig. 6(a) to Fig. 6(b). Table 1 lists the physical dimensions of the initial structure, ES only optimization, and ES with suppressing ripples (SR).

The positive effect of the proposed method is clearly seen, when the s-parameters of $S_{21}$ and $S_{11}$ as the frequency responses are plotted and compared between of the initial structure, genetic algorithm (GA) optimization, ES only optimization, and ES with suppressing ripples. The insertion loss of the initial filter is higher than $3 \mathrm{~dB}$ with many ripples, and its stopband attenuation is relatively very small as in Fig. 7(a). The ES and GA can somewhat improve the frequency response by decreasing the ripple level, and make a wider stopband. Nevertheless, only the ES and GA have still the maximum ripples that are $0.78 \mathrm{~dB}$ as in Fig. 7(a). But the ripples can be completely removed by the modified ES optimization. Using the physical dimensions in the third column of Table 1, no ripple is observed from $\mathrm{S}_{21}$ in Fig. 7(b). Besides, the stopband and return loss are

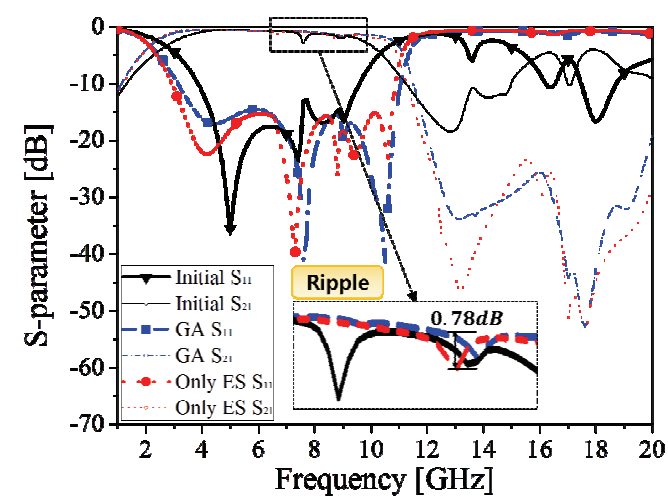

(a)

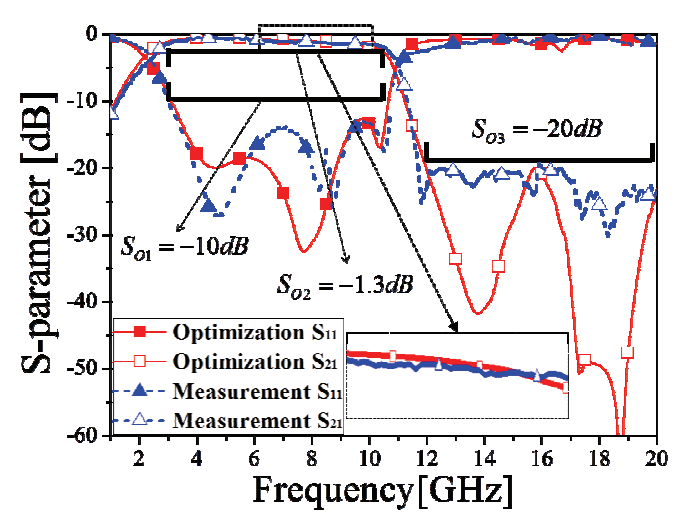

(b)

Fig. 7. $S_{21}$ and $S_{11}$ of the filters before and after optimization (a) Initial, GA and only the ES (b) Comparison of measurement and result calculated by the proposed ES

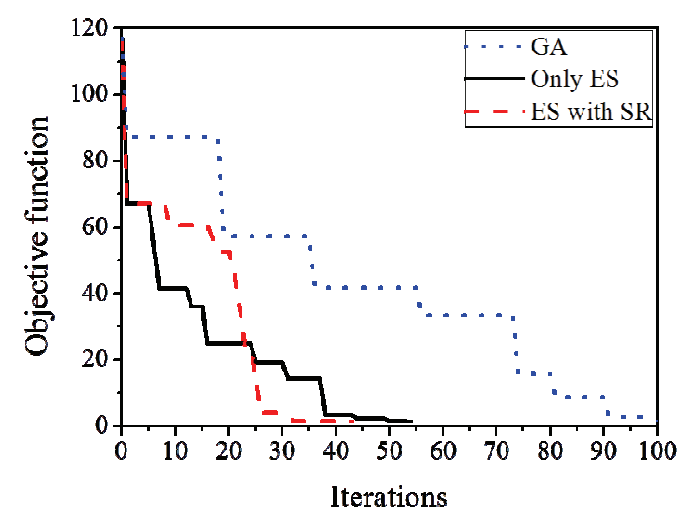

Fig. 8. Convergence of the objective function

satisfactory as shown in Fig. 7(b).

Another advantage is accelerating the optimization. This is presented by comparing the convergence of the cost functions of the conventional ES, GA and the proposed ES. The convergence takes place after the 43th iteration in the proposed ES approach. But, the objective function of the conventional ES starts to converge from the 54th iteration. This is interpreted that the proposed method with the ripple suppression achieves the goals faster than the conventional 


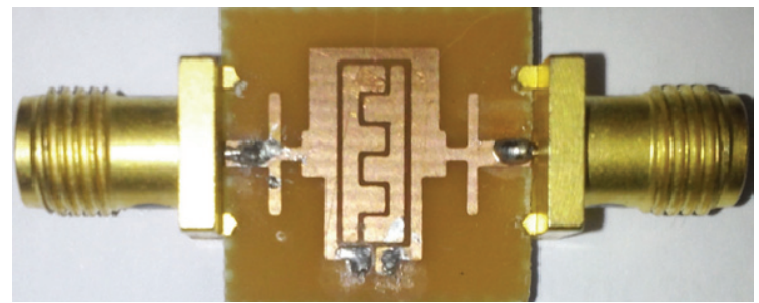

Fig. 9. The photograph of fabricated UWB filter

technique with the ripples disturbing the steps to reach the targets. Finally, the optimized CRLH UWB filter is fabricated. Its photograph is given in Fig 9.

The total size of the filter is, the size of $15 \times 14 \mathrm{~mm}$. The s-parameters of the proposed SI-combined CRLH UWB filter are measured. The measurement is in a fairly good agreement with the simulation, and the outcome shows the the $3 \mathrm{~dB}$ bandwidth from 2.7 to $10.8 \mathrm{GHz}$, the flat insertionloss less than $1 \mathrm{~dB}$, an acceptable return-loss, and the wide stopband of 12 20GHz as shown in Fig. 7.

\section{Conclusion}

We proposed an efficient optimization design method of a compact UWB filter by using the modified ES algorithm. Compared to the general ES, the proposed ES successfully mitigated the ripple in the passband and accelerate the optimization. The optimized filter was prominently changed to the shape, which includes the SI and CRLH-TL. The optimized filter has the compact size of $15 \times 14 \mathrm{~mm}$, the $3 \mathrm{~dB}$ bandwidth from 2.7 to $10.8 \mathrm{GHz}$ the flat insertionloss less than $1 \mathrm{~dB}$, an acceptable return-loss, and the wide stopband of 12 20GHz. The proposed design scheme will be applicable to any wideband and compact filters.

\section{Acknowledgement}

This research was supported by Basic Science Research Program through the National Research Foundation of Korea (NRF) funded by the Ministry of Education, Science and Technology (2012R1A1A2007758) and in part by the Chung-Ang University Asian Young Scientist Scholarship. Also we wish to thank Prof. Sungtek Kahng for help.

\section{References}

[1] H. Wang, L. Zhu and W. Menzel, "Ultra-Wideband Bandpass Filter with Hybrid Microstrip/CPW Structure," IEEE Microwave And Wireless Component Letters, vol. 15, no. 12, pp. 844-846, December 2005.

[2] C.-L. Hsu, F.-C. Hsu, and J.-T. Kuo, "Microstrip Bandpass Filters for Ultra-Wideband (UWB) Wireless Communications," IEEE MTT-S Intl. Dig., pp. 675-
678, June 2005.

[3] Christophe Caloz, Tatsuo Itoh, Electromagnetic Metamaterials: Transmission Line Theory and Microwave Applicaions, WILEY-INTERSCIENCE, John-Wiley \& Sons Inc., Hobokin, NJ 2006.

[4] J. Ju, S. Kahng, "A compact UWB bandpass filter using a center-tapped composite right/left-handed transmission-line zeroth-order resonator" Microwave and Optical Technology Letters, vol. 53, Issue 9, pp. 1974-1976, Sep. 2011.

[5] S. Kahng, et al., "A Center-Tapped CRLH ZOR UWB Bandpass Filter with Improved Stopband", MICROWAVE JOURNAL, vol. 55, Issue 6, pp. 86 86, June. 2012.

[6] S. Kahng, et al., "Compact metamaterial zeroth-order resonator bandpass filter for a UHF band and its stopband improvement by transmission zeros", IETMicrowaves, Antennas \& Propagation, Vol. 5, Issue 10, pp. 1175 1181, July. 2011.

[7] B. Lee, S. Kahng, "A compact UWB bandpass filter using a center-tapped composite right/left-handed transmission-line zeroth-order resonator" Microwave and Optical Technology Letters, vol. 53, Issue 9, pp. 1974-1976, Sep. 2011.

[8] S. Kahng, et al., "Compact UWB Bandpass Filter as Cascaded Center-Tapped CRLH Transmission-Line ZORs for Improved Stopband", Journal of Electronical Engineering \& Technology, vol. 8, no. 2, pp. 371-375, Aug. 2015.

[9] David M. Pozar, Microwave Engineering 3rd Edition, John Wiley \& Sons, Inc., Wiley publishing company 2005.

[10] K.-T. Kim, J.-H. Ko, K. Choi, H.-S. Kim, “Optimum Design of Wideband Bandpass Filter with CSRRLoaded Transmission Line Using Evolution Strategy", IEEE Trans. on Magnetics, Vol. 48, No. 2, pp. 811814, Feb. 2012.

[11] K. C. Gupta, R. Garg, I. Bahl, and P. Bhartia, Microstrip Lines and Slotlines, Artech House, Inc., Norwood, MA 1996.

[12] G. Matthaei, L. Young, and E. M. T. Jones, Microwave Filters, Impedance-Matching Networks, and Coupling Structures, Artech House, Inc., 1980.

[13] T. Back, Evolutionary Algorithms in Theory and Practice. Oxford, U.K.: Oxford Univ. Press, 1996.

[14] HFSS, Version 13, Ansoft Corporation LLC, 225 West Station, Square Suite 200, Pittsburgh, PA 15219-1119, 2011. 


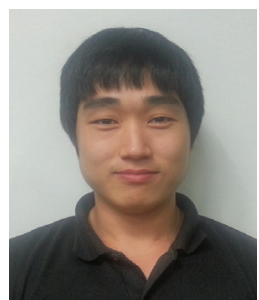

Seung-Hun Oh was born in Buan, South Korea. He received his B.S. degree in electrical and electronics engineering from Chung-Ang University, South Korea in 2013. He is working toward the M.S. degree in electrical and electronics engineering at the same university. His interests include optimize design of passive component, equivalent circuit model, analysis on RF components, and metamaterial.

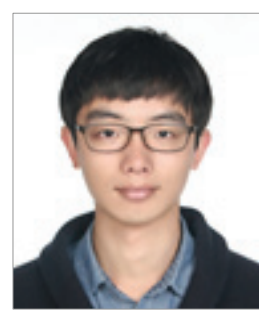

CHAO WU was born in Suzhou, China. He received his B.S. degree in electrical and electronics engineering from Konkuk University, South Korea in 2014. He is working toward the M.S. degree in electrical and electronics engineering at Chung-Ang University, South Korea. His interests include analysis on RF component, optimal design of passive component.

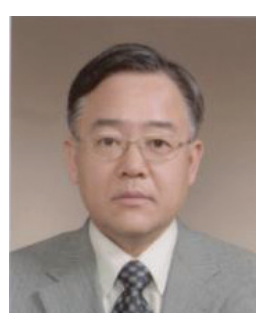

Tae Kyung Chung He received the B.S., M.S., and Ph.D. degrees in electrical engineering from Seoul National University in 1981, 1983, and 1987, respectively. From 1986 to 1987 , he worked at Daewoo Heavy Industries as a senior researcher. Since 1988, he has been with the School of Electrical and Electronics Engineering at Chung Ang University in Korea as a professor. In 1991, he was with Harvey Mudd College in California as a visiting scholar. His main research fields are design of electric motors and actuators.

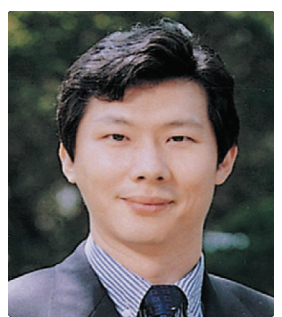

Hyeong-Seok Kim was born in Seoul, South Korea in 1962. He received his B.S., M.S., and Ph.D. degrees from the Department of Electrical Engineering at Seoul National University, Korea in 1985, 1987, and 1990, respectively. From 1990 to 2002, he worked with the Division of Information Technology Engineering, Soonchunhyang University in Asan, Korea. He was a Visiting Professor of Electrical Computer Science Engineering at the Rensselaer Polytechnic Institute in Troy, New York in 1997. He transferred to the School of Electrical and Electronics Engineering, Chung-Ang University, Seoul, Korea as a professor in 2002. His current research interests include numerical analysis of electromagnetic field and waves, optimal and robust design of passive components for wireless communication, RFID applications, wireless power transmission, and electromagnetic education. He is the corresponding author of this paper. 